\title{
Ageing and high rate studies on resistive Micromegas at the CERN Gamma Irradiation Facility
}

\author{
E. Farina ${ }^{* a}$, B. Alvarez Gonzalez ${ }^{b}$, J. Bortfeldt ${ }^{c}$, P. lengo ${ }^{c}$, J. Samarati ${ }^{c}$, G. \\ Sekhniaidze $^{d}$, O. Sidiropoulou ${ }^{c}$, J. Wotschack ${ }^{c}$ \\ ${ }^{a}$ University of Pavia and INFN \\ ${ }^{b}$ University of Oviedo \\ ${ }^{c}$ CERN \\ ${ }^{d}$ INFN Napoli \\ E-mail: edoardo.maria.farina@cern.ch
}

\begin{abstract}
Two resistive-strip bulk Micromegas detectors have been installed in the Gamma Irradiation Facility at CERN in 2015. The primary goal was to assess the performance of the detectors after long term irradiation. This study is particularly relevant in view of the adoption of the Micromegas technology for future upgrades, as in the ATLAS inner muon system. In this region the expected accumulated charge in 10 years of HL-LHC operation has been estimated to be $0.2 \mathrm{C} / \mathrm{cm}^{2}$. After 3 years of continuous irradiation more than $0.3 \mathrm{C} / \mathrm{cm}^{2}$ have been accumulated, allowing to draw clear conclusions concerning ageing effects on the detectors. During this period, the detector currents have been monitored and the performance of the detectors has been studied by combining the photon background with muon beam available at the facility. In particular, the spatial resolution and detector gain have been studied up to about $70 \mathrm{kHz} / \mathrm{cm}^{2}$.
\end{abstract}

The 39th International Conference on High Energy Physics (ICHEP2018)

4-11 July, 2018

Seoul, Korea

*Speaker. 


\section{Introduction}

Ageing properties of gaseous detectors and the capability of providing high performance at high particle rate are key elements in the development of the detectors for future high energy experiments.

Our study concerns two bulk resistive Micromegas detectors [1], which have been installed in the Gamma Irradiation Facility (GIF++)[2] at CERN in 2015, where a very intense photon irradiation can be combined with a muon beam. The photon background is produced by a $14 \mathrm{TBq}$ Cesium source, whose energy spectrum mimics the expected background in a typical LHC environment. The intensity of the source can be partially shielded, attenuating the particle rate reaching the detectors. The detectors have been placed at a distance from the source to accelerate the charge accumulation of about 5 times with respect to the expected rate in the final experiment, aiming to collect $0.2 \mathrm{C} / \mathrm{cm}^{2}$, equivalent to 10 years of High Luminosity LHC operations. The photon rate simulated without any attenuation shield in the chosen position is of about $5 \cdot 10^{7}$ photons $/ \mathrm{cm}^{2}$ [3], for a measured interaction rate of about $120 \mathrm{kHz} / \mathrm{cm}^{2}$. The two detectors consist of standard resistive Micromegas [1] characterized by a readout anode plane made of $400 \mu \mathrm{m}$ pitch metallic readout electrodes covered by a $50 \mu \mathrm{m}$ Kapton ${ }^{\circledR}$ layer on top of which resistive strips are deposited.

Amplification gap is of $100 \mu \mathrm{m}$, while the drift is $5 \mathrm{~mm}$. The resistivity of the resistive pattern is of few $\mathrm{M} \Omega / \mathrm{cm}^{2}$. The stainless steel mesh consists of $18 \mu \mathrm{m}$ diameter wires with $45 \mu \mathrm{m}$ pitch. Since 2015 the detectors have been constantly monitored, performing dedicated test campaigns to assess the detectors performance. The gas used for all the tests is an $\mathrm{ArCO}_{2}$ (93:7); the readout system adopted is based on APV-25 front-end ASICs [4] and RD51 Scalable Readout System (SRS)[5].

\section{High rate studies}

The detector performance has been measured with a muon beam as a function of the background rate. Muons tracks have been reconstructed in the photon background by means of the Hough transform technique [6]. The spatial resolution has been obtained from the residual distribution of the observed cluster position with respect to the expected one as obtained from external tracking chambers. Results are reported in Fig. 1 (a), where no degradation of the resolution is shown up to $70 \mathrm{kHz} / \mathrm{cm}^{2}$. The cluster charge distribution has been also investigated, by considering the most probable value (MPV) of the Landau fit, to highlight a possible gain reduction at high rate. Figure 1 (b) reports the results, without any significant reduction up to about $40 \mathrm{kHz} / \mathrm{cm}^{2}$ and a reduction of about $10 \%$ at $70 \mathrm{kHz} / \mathrm{cm}^{2}$. These results are in agreement with the expected performance of this detector typology, designed to operate at about $15 \mathrm{kHz} / \mathrm{cm}^{2}$.

\section{Long term ageing}

From May 2015, to December 2017 more than $0.3 \mathrm{C} / \mathrm{cm}^{2}$ have been accumulated on both the detectors, called T5 and T8 in the following, largely exceeding our initial goal. The detector performance has been measured three times, one at the beginning of the irradiation, one after accumulating $0.2 \mathrm{C} / \mathrm{cm}^{2}$, and one at the end of the irradiation. In particular the ageing of a detector 


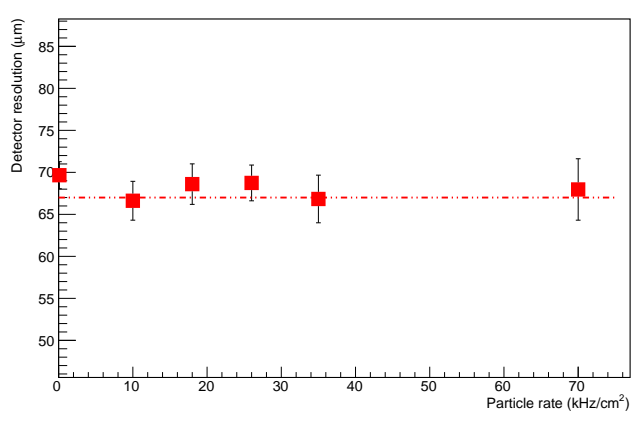

(a)

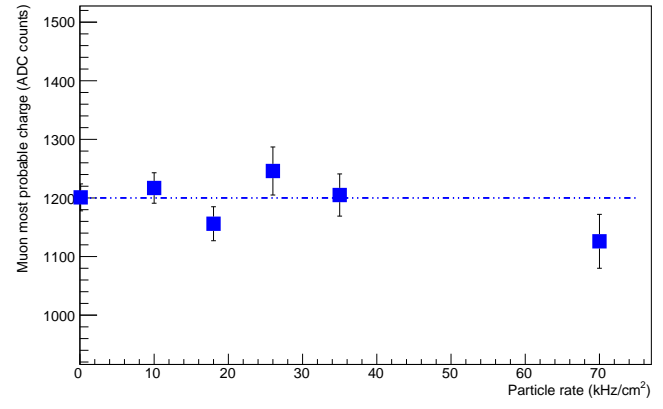

(b)

Figure 1: Resolution (a) and most-probable-value of muon charge as a function of the particle rate.

could lead to a reduction of the amplification gain, and a shift of the working point at which the chamber needs to be operated to be efficient. The gain of both the detectors has been measured by means of an Iron source, removing the detectors from the facility, and by measuring the efficiency to muons as a function of the amplification field. In this case the efficiency is defined as the presence of a muon cluster in proximity of the expected position as defined by tracking chambers. The gas gain and efficiency for the T5 chamber are reported in Fig. 2 (a and b respectively) for the three measurement campaigns. Both efficiency and gain measurements are not corrected for environmental parameters, which can account for the small difference observed, in particular at lower voltage, when the cluster charge is smaller. For what concerns the efficiency, no shift of the working point of the detector has been observed. In conclusion no sign of detector ageing has been observed from these measurements. The same conclusions have been be also derived for the other detector, not reported here for space limitation.

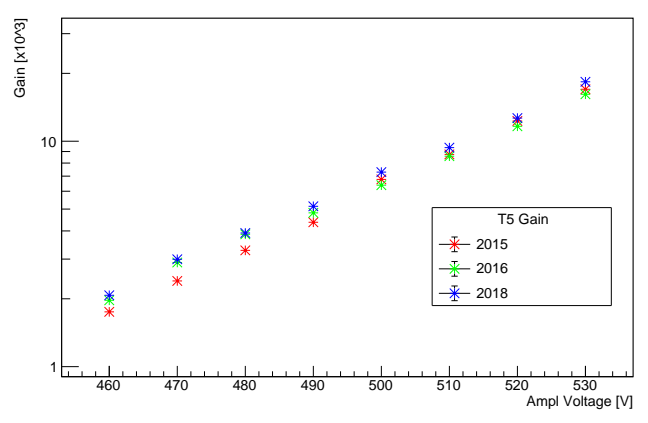

(a)

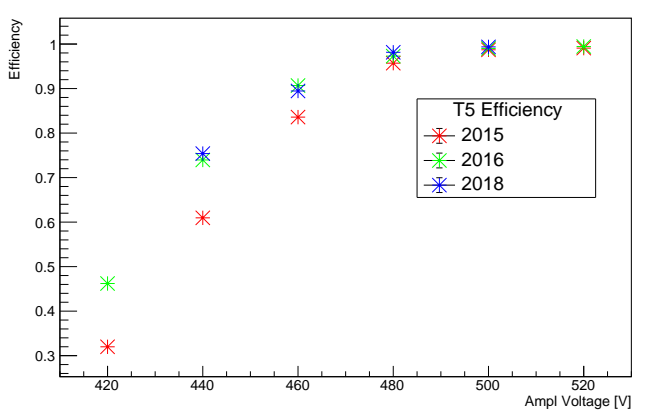

(b)

Figure 2: Gas gain and efficiency for T5 as a function of the amplification voltage for the three measurements compared.

An evaluation of the detector behaviour throughout the time can also be performed by looking at the detector current as a function of the time. Possible changes of the relative position of the detectors with respect to the source and of the shielding settings have to be taken into account to assure that any change in the current is due to a real effect in the detector. In Fig. 3a the integrated 
charge as a function of time is reported for the T5 detector. Figure $3 \mathrm{~b}$ shows the current trend as a function of time. On the $\mathrm{X}$ axis the different irradiation periods in which the long term study has been segmented, and in which no changes in the environmental conditions took place, are reported. The average duration of a single period is about 2 days. The current has been then normalized to the initial value. Only small variations have been observed, suggesting, also in this case, that no ageing effects have taken place. For space reasons only results from one detector have been reported; similar results have been obtained for the second one.

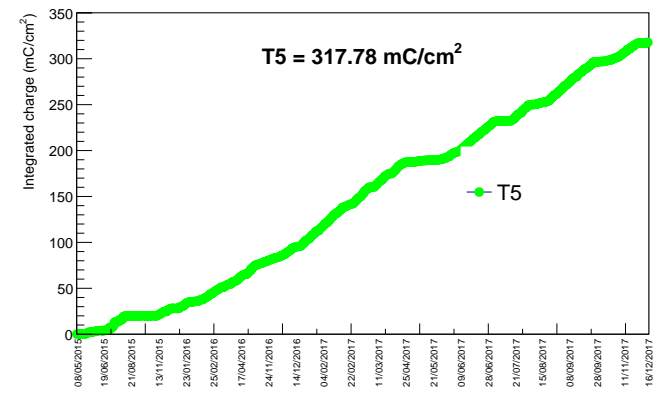

(a)

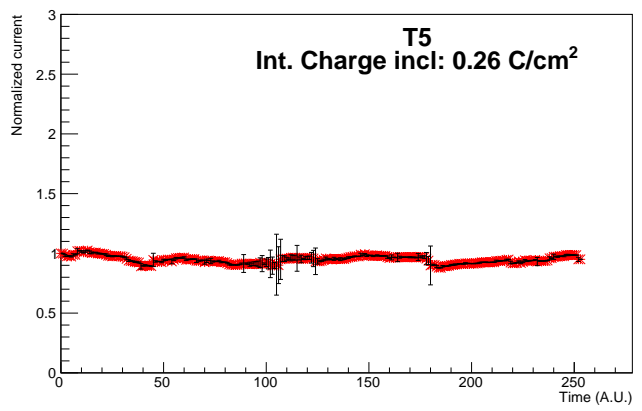

(b)

Figure 3: Integrated charge (a) and current trend (b) for T5 detector as a function of time.

\section{Conclusions}

Two bulk Micromegas have been installed in the GIF++ facility for high rate and long term ageing studies. Performance measured as a function of the rate has clearly shown that these detectors can work at the rate of about $40 \mathrm{kHz} / \mathrm{cm}^{2}$ without any performance degradation. Both detectors have accumulated more than $0.3 \mathrm{C} / \mathrm{cm}^{2}$ equivalent to more than 10 years of High luminosity LHC operations at a distance from the interaction point typical of the innermost ATLAS muon detector system. Measurements of the gas gain, efficiency and current stability showed that no ageing effects have been observed.

\section{References}

[1] T. Alexopoulos et al., Nucl. Instrum. Meth. A 640 (2011) 110.

[2] M. R. Jaekel et al., CERN GIF++, PoS (TIPP2014) 102

[3] D. Pfeiffer et al., https://arxiv.org/abs/1611.00299

[4] M.Raymond et al., IEEE Nucl. Sci. Symp. Conf. Rec 2 (2000)

[5] S. Martoiu et al., JINST 8 C03015 (2013)

[6] R. Fisher, S. Perkins, A. Walker and E. Wolfart, Hough Transform, 2003 URL: http://homepages.inf.ed.ac.uk/rbf/HIPR2/hough.htm 